\title{
Recubrimiento comestible a partir del mucilago del café (Coffea arabica) para la conservación de manzanas
}

\section{Edible coating from coffee mucilage (Coffea arabica) for the preservation of apples}

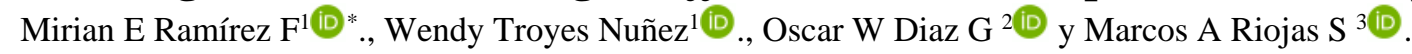

\section{RESUMEN}

El objetivo de la investigación fue determinar la formulación de un recubrimiento comestible (RC) a partir del aprovechamiento del mucílago del café y su aplicación en la conservación de manzanas Red Delicious. Se realizó formulaciones de mucilago y gelatina en las proporciones (F1: 94 y 6\%; F2: 92 y 8\%; F3: 90 y 10\%; F4: 88 y 12\%). La formulación fue mediante el método de inmersión de la fruta durante un minuto, se secaron a temperatura ambiente y se almacenaron por 15 días. En este periodo, se evaluó características fisicoquímicas (pérdida de peso, ${ }^{\circ}$ Brix, $\mathrm{pH}$, acidez titulable, e índice de madurez), se realizó un análisis microbiológico y sensorial al final del tiempo de almacenamiento. Las características fisicoquímicas y sensoriales se evaluaron mediante un análisis de varianza y prueba Tukey 95\%. Los resultados en la aplicación del RC en sus diferentes formulaciones ayudaron a controlar las características fisicoquímicas y su aplicación es microbiológicamente segura. Por último, el análisis sensorial mostró que la formulación F1 presenta mejor aceptación, según la Prueba de Tukey a un nivel de confianza del 95\%. Esta formulación no altera las características sensoriales, retrasa la maduración y deterioro, preserva sus atributos comerciales y alimenticios.

Palabras clave: Recubrimiento comestible, manzana red delicious, análisis sensorial

\begin{abstract}
The aim of the research was to determine the formulation of an edible coating (EC) from coffee mucilage The objective of the research was to determine the formulation of an edible coating (EC) from the use of coffee mucilage and its application in the preservation of Red Delicious apples. Formulations of mucilage and gelatin were made in the following proportions (F1: 94 and 6\%; F2: 92 and 8\%; F3: 90 and 10\%; F4: 88 and 12\%). The formulation was made by immersion of the fruit for one minute, dried at room temperature and stored for 15 days. During this period, physicochemical characteristics were evaluated (weight loss, ${ }^{\circ}$ Brix, $\mathrm{pH}$, titratable acidity, and maturity index), and a microbiological and sensory analysis was performed at the end of storage. The physicochemical and sensory characteristics were evaluated by analysis of variance and Tukey 95\% test. The results in the application of EC in its different formulations helped to control the physicochemical characteristics and its application is microbiologically safe. Finally, the sensory analysis showed that the F1 formulation presents better acceptance, according to the Tukey test at a 95\% confidence level. This formulation does not alter the sensory characteristics, delays ripening and spoilage, and preserves its commercial and nutritional attributes.
\end{abstract}

Keywords: Edible coating, red delicious apple, sensory analysis.

DOI: https://doi.org/10.37787/pakamuros-unj.v9i4.233

Recibido: 14/04/2021. Aceptado: 29/10/2021

* Autor para correspondencia

1. Universidad Nacional de Jaén, Perú. Email: merf2910@gmail.com; wendytroyes@gmail.com

2. Universidad Nacional Autónoma de Tayacaja, Perú. Email: oscarw003@yahoo.com.br

3. Universidad Nacional Pedro Ruiz Gallo, Perú. Email: mriojassandoval@gmail.com 


\section{INTRODUCCIÓN}

El café es el principal producto agrícola de exportación en el Perú, a pesar de su importancia reconocida, el impacto ambiental ocasionado por los residuos generados es alto. Estos desechos representan alrededor de 95 \% del total del peso del grano, son una fuente importante de contaminación y estos residuos se han aprovechado como materia prima para la producción de piensos, bebidas, vinagre, biogás, cafeína, pectina y azucares (Puertas, 2013).

Entre los desechos del café destaca la pulpa, la cual cuenta con mucílago, compuesto heterogéneo con propiedades viscosantes y gelificantes; razón por la cual se ha determinado en diversos estudios su utilización en formulaciones de productos alimenticios. Del mucilago de café, se puede obtener, pectinas, azucares naturales, compuestos antioxidantes y flavonoides (Rathinavelu \& Graziosi, 2005).

Para obtener un alimento saludable, incluyen frutas y vegetales frescos y saludables puesto que les proporcionan nutrientes necesarios. Las frutas y vegetales son una fuente importante de vitaminas, minerales, y otros compuestos bioactivos que presentan propiedades antioxidantes (Corbo et al, 2015). Para la conservación de frutas y vegetales, se utilizan películas y recubrimientos comestibles como alternativa prometedora para mejorar su calidad durante su procesado y conservación (Ancos, et al. 2015). Los recubrimientos y películas comestibles están constituidos por finas películas de polímeros naturales (polisacáridos, proteínas animales y vegetales, lípidos) biodegradables, por lo que es una tecnología respetuosa con el medio ambiente que responde a la demanda creciente por parte de los consumidores de alimentos naturales, seguros, saludables y obtenidos mediante un procesado mínimo (Rodriguez, 2016). Los recubrimientos comestibles se obtienen a partir de mucilagos de plantas y vegetales. Los mucílagos son polisacáridos heterogéneos, formados por diferentes azucares y en general ácidos urónicos y se caracterizan por formar disoluciones coloidales viscosas (geles en agua) (Rojas, 2006). Allegra et al. (2016) emplearon el mucílago del nopal (Opuntia ficus-indica) para el desarrollo de un RC aplicado el kiwi de IV gamma almacenado en refrigeración durante 12 días. Al final del análisis, los productos recubiertos presentaron mayor firmeza que los sin recubrir, además no se reportaron cambios en la aceptación del sabor de la muestra. Fai, et al. (2016), reportaron el uso y revalorización de residuos de industrias alimenticias, en la preparación de un RC a partir de harina de residuos de frutas y vegetales previamente tratados, para la aplicación en zanahoria de IV gama rallada (ZR) y cortada (ZC). Se reportaron que las muestras de ZR presentaron mejor coloración característica del producto que las de ZC durante el tiempo de almacenamiento. Además, al comparar las muestras de ZR tratadas y el control, se evidenciaron menores pérdidas de peso en el producto recubierto. 
Por lo expuesto, se desconoce a nivel científico la formulación de un RC a partir del aprovechamiento del mucílago del café y su aplicación en la conservación de manzanas Red Delicious. En ese sentido se planteó como pregunta de la investigación ¿Cuál es la formulación de un RC para su aplicación en la conservación de manzanas Red Delicious? En este sentido, el objetivo de esta investigación fue determinar la formulación de un recubrimiento comestible a partir del aprovechamiento del mucílago del café y su aplicación en la conservación de manzanas Red Delicious.

\section{MATERIALES Y MÉTODOS}

\section{Lugar de ejecución}

La investigación se realizó en los laboratorios de la Carrera de Ingeniería de Industrias Alimentarias de la Universidad Nacional de Jaén.

\section{Análisis fisicoquímico del mucilago del café}

El análisis del pH se determinó con ayuda del potenciómetro y los sólidos solubles o grados ${ }^{\circ}$ Brix, mediante la aplicación de 1-2 gotas del mucilago en el prisma del refractómetro Mettler y se cerró la tapa para que se mezcle homogéneamente; se sostuvo el refractómetro bajo la luz solar y se pudo ver la escala a través del ocular.

\section{Análisis fisicoquímico del recubrimiento comestible}

El análisis de $\mathrm{pH}$, solidos solubles y el porcentaje de humedad se obtuvo mediante el método gravimétrico (se calentó la estufa x 15 min a $105^{\circ} \mathrm{C}$, se pesaron 5 g de muestra en una capsula y se colocó en el desecador por $10 \mathrm{~min}$ ), consecutivamente se colocó la muestra en la estufa por $2 \mathrm{~h}$ a $105^{\circ} \mathrm{C}$, luego se sacó la muestra y se colocó en el desecador hasta que enfrié, se pesó. El contenido en agua de la muestra se calculó por diferencia de peso y se expresa en porcentaje de humedad (g de $\mathrm{H}_{2} \mathrm{O} / 100 \mathrm{~g}$ de muestra) ver Ecuación (1).

$$
\% \text { Humedad }=\frac{\text { Peso de muestra }}{\text { Apeso de la muestra humeda }} \times 100
$$

\section{Determinación del gramaje}

Se cortaron tres muestras por formulación, las cuales presentaron las mismas dimensiones (largo $\mathrm{x}$ ancho: $2 \mathrm{~cm}$ x $2 \mathrm{~cm}$ ), se pesaron las fracciones de recubrimiento y se calculó el valor promedio de las tres muestras explicando los resultados en g/cm², Ecuación (2).

$$
G=\frac{P}{A}
$$

Donde $G=$ Gramaje $\left(\mathrm{g} / \mathrm{cm}^{2}\right) ; p=$ Peso medio (g); $A=$ área de la muestra $\left(\mathrm{cm}^{2}\right)$ 


\section{Determinación del espesor}

El espesor del recubrimiento comestible se midió con un vernier o pie de rey (calibrador Vernier Caliper, Size: 0 - $150 \mathrm{~mm} / 6$ ”, kamasa profesional), haciéndose cuatro medidas de los recubrimientos de acuerdo a su respectiva formulación. El espesor del recubrimiento se calculó como la media aritmética de las 4 medidas obtenidas.

\section{Análisis fisicoquímicos de manza red delicious}

El analisis fisicoquimco de la manzana red delicios se realizo mendiante el Método 947.05 A.O.A.C. 2010 y el metodo directo (Tabla 1).

Tabla 1. Análisis fisicoquímico de la manzana Red Delicious

\begin{tabular}{llc}
\hline Análisis/ Manzanas & Método & Nombre del equipo \\
\hline Acidez titulable & Método 947.05 A.O.A.C. & - \\
& $(2010)$ & \\
Análisis de pH & Directo & $\mathrm{pH}-\mathrm{metro}$ \\
Solidos solubles & Directo & ${ }^{\mathrm{a}} \mathrm{Bx}$ \\
Índice de madurez & Directo & \\
\hline
\end{tabular}

\section{Determinación de la Acidez}

Se pesaron 5 gr de muestra y se disolvió con $50 \mathrm{ml}$ de agua destilada en un matraz Erlenmeyer y se añadió 3 gotas de fenolftaleína. Se hizo una titulación rápida con $\mathrm{NaOH} 0.1 \mathrm{~N}$, Ecuación (3).

$$
\% \text { Acidez }=100\left(\frac{N \cdot V \cdot p e X}{W}\right)
$$

Donde: $N=$ Normalidad de la solucion $\mathrm{NaOH} ; \mathrm{V}=\mathrm{ml}$ de $\mathrm{NaOH}$ gastado en la titulación; $p e X=$ Peso mili equivalentes del ácido organico $X ; W=$ Peso de la muestra.

\section{Índice de madurez}

Para determinar el índice de madurez se realizó mediante la Ecuación (4).

$$
\text { índice de madurez }=\frac{{ }^{\circ} \text { Brix }}{\text { Acidez }}
$$

\section{Evaluación microbiológica}

Se realizó en el laboratorio de Microbiología de la Facultad de Tecnología Médica de la Universidad Nacional de Jaén. Se usó el método de ensayo de Petri Film, determinando los tipos de microorganismos (Aeróbios totales, Coliformes Totales, E. coli, Mohos y Levaduras). Donde el recuento de Coliformes Totales y E. coli está basada en la Técnica PETRIFILM® A.O.A.C Official Method 991.14 o 998.08; 
el recuento de Aeróbios Mesófilos, basada en la Técnica PETRIFILM® A.O.A.C Official Method 990.12; y finalmente el recuento de Mohos y Levaduras, bajo la Técnica PETRIFILM® A.O.A.C Official Method 997.02.

\section{Evaluación sensorial}

Al final del tiempo de almacenamiento se realizó el análisis sensorial, utilizando pruebas afectivas (Prueba de aceptación, 30 panelistas) donde el panelista expresó su nivel de agrado, aceptación y preferencia del producto. A cada descriptor se le asignó un rango o intervalo numérico, y el panelista identificó en este intervalo. La escala puede ser estructurada (con valores intermedios en la escala) o no estructurada (que contiene solo los valores extremos del intervalo).

\section{Evaluación Estadística}

Para el análisis estadístico de las muestras, se utilizó un ANOVA y las Pruebas de Rangos Múltiples, para indicar cuales medias son significativamente diferentes de otras, se utilizó el Método: 95.0 \% Tukey HSD. Los datos se analizaron con el programa estadístico SPSS.

\section{RESULTADOS}

\section{Análisis fisicoquímico de mucilago del café}

Tabla 2 y 3, muestran los resultados del mucilago de café extraídos después de 42 horas de fermentación así como la caracterización del recubrimiento comestible en sus diferentes formulaciones. Donde los valores de pH del mucílago fermentado entre 3.7 y 4.1 son adecuados y seguros para interrumpir la fermentación y lavar el café.

Tabla 2. Análisis fisicoquímico de mucílago del café

\begin{tabular}{cc}
\hline Análisis & Resultado \\
\hline${ }^{\circ} \mathrm{Brix}$ & 10.5 \\
$\mathrm{pH}$ & 3.9 \\
\hline
\end{tabular}

Tabla 3. Análisis fisicoquímico del recubrimiento comestible en sus diferentes formulaciones

\begin{tabular}{lcccc}
\hline Análisis & F1 & F2 & F3 & F4 \\
\hline $\mathrm{pH}$ & 4.2 & 4.3 & 4.4 & 4.5 \\
${ }^{\circ}$ Brix & 14.5 & 15 & 17.8 & 19 \\
Humedad & $11.37 \%$ & $10.83 \%$ & $10.26 \%$ & $9.76 \%$ \\
Gramaje $\left(\mathrm{g} / \mathrm{cm}^{2}\right)$ & 7.5 & 10.25 & 17.25 & 27.75 \\
Espesor $(\mathrm{um})$ & 2.6 & 5.5 & 10.5 & 12.5 \\
\hline
\end{tabular}

F1; F2; F3 y F4=diferentes formulaciones

En la Tabla 4, se evidencia que los resultados se ajustan a la ficha técnica utilizada por el Organismo Supervisor de las Contrataciones del Estado (OCSE), para la comercialización de manzanas red 
delicious frescas. El grado ${ }^{\circ}$ Brix, estuvo dentro de los rangos establecidos por dicha norma (10 - 13). Además, las características sensoriales, se ajustan a dicha ficha técnica.

Tabla 4. Evaluación fisicoquímica de la manzana Red Delicious

\begin{tabular}{lc}
\hline Análisis & Resultado \\
\hline${ }^{\circ}$ Brix & 11 \\
$\mathrm{pH}$ & 4.6 \\
Acidez titulable & $0.20 \%$ \\
Índice de madurez & 55 \\
\hline
\end{tabular}

En la Tabla 5, se observan las características organolépticas del sabor, olor, color y textura de la manzana red delicious

Tabla 5. Evaluación sensorial de las manzanas Red Delicious

\begin{tabular}{lc}
\hline $\begin{array}{c}\text { Característica } \\
\text { organoléptica }\end{array}$ & Evaluación \\
\hline Sabor & Pulpa blanda dulce y aromática \\
Olor & Característico \\
Color & Rojo brillante \\
Textura & Firme \\
\hline
\end{tabular}

\section{Evaluación fisicoquímica durante el periodo de almacenamiento}

En la Tabla 6 y 7, se muestra los resultados de la evaluación porcentual de pérdida de peso en manzanas Red Delicious con RC en diferentes formulaciones.

Tabla 6. Evaluación porcentual de pérdida de peso en manzanas Red Delicious con RC en diferentes formulaciones

\begin{tabular}{rrrrrrr}
\hline \multirow{2}{*}{ Días } & \multicolumn{7}{c}{ Pérdida de peso (\%) } \\
\cline { 2 - 7 } & Patrón (P) & F1 & \multicolumn{2}{c}{ F2 } & F3 & F4 \\
\hline 0 & $0 \%$ & $0 \%$ & $0 \%$ & $0.00 \%$ & $0 \%$ \\
2 & $0.39 \%$ & $0.36 \%$ & $0.35 \%$ & $0.32 \%$ & $0.31 \%$ \\
4 & $0.77 \%$ & $0.71 \%$ & $0.68 \%$ & $0.62 \%$ & $0.61 \%$ \\
6 & $1.14 \%$ & $1.05 \%$ & $0.97 \%$ & $0.93 \%$ & $0.89 \%$ \\
8 & $1.50 \%$ & $1.32 \%$ & $1.23 \%$ & $1.17 \%$ & $1.16 \%$ \\
10 & $1.85 \%$ & $1.55 \%$ & $1.47 \%$ & $1.40 \%$ & $1.43 \%$ \\
12 & $2.18 \%$ & $1.78 \%$ & $1.71 \%$ & $1.63 \%$ & $1.68 \%$ \\
14 & $2.50 \%$ & $2.00 \%$ & $1.95 \%$ & $1.84 \%$ & $1.81 \%$ \\
\hline
\end{tabular}


Tabla 7. Registro de ${ }^{\circ}$ Brix de manzana Red Delicious con RC en diferentes formulaciones

\begin{tabular}{cccccc}
\hline & \multicolumn{5}{c}{${ }^{\mathbf{0}}$ Brix } \\
\cline { 2 - 6 } Días & Patrón & F1 & F2 & F3 & F4 \\
\hline 0 & 11 & 11 & 11 & 11 & 11 \\
2 & 11 & 11 & 11 & 11 & 11 \\
4 & 12.2 & 11 & 11.5 & 11.5 & 11.5 \\
6 & 12.6 & 11.5 & 11.5 & 11.5 & 11.5 \\
8 & 12.6 & 12 & 11.8 & 11.8 & 12 \\
10 & 12.8 & 12.4 & 12 & 12 & 12 \\
12 & 13 & 12.4 & 12.2 & 12.5 & 12.3 \\
14 & 13.5 & 13 & 12.5 & 12.5 & 12.3 \\
\hline
\end{tabular}

Tabla 8, se puede evidenciar que el $\mathrm{pH}$ se vio afectado por el uso de recubrimiento comestibles, y pudo estar relacionado con la disminución de la senescencia del fruto, evitando que durante la maduración de la manzana algunos fragmentos de pectinas se liberen desde la pared celular y se unan a los polifenoles, lo cual incrementa los valores de $\mathrm{pH}$.

Tabla 8. Registro de pH de manzanas Red Delicious con RC en diferentes formulaciones

\begin{tabular}{cccccc}
\hline Días & \multicolumn{5}{c}{$\mathbf{p H}$} \\
\cline { 2 - 6 } & Patrón & F1 & F2 & F3 & F4 \\
\hline 0 & 4.41 & 4.41 & 4.41 & 4.41 & 4.41 \\
2 & 4.42 & 4.42 & 4.42 & 4.42 & 4.41 \\
4 & 4.5 & 4.43 & 4.44 & 4.44 & 4.43 \\
6 & 4.52 & 4.46 & 4.46 & 4.45 & 4.43 \\
8 & 4.56 & 4.5 & 4.48 & 4.48 & 4.5 \\
10 & 4.6 & 4.58 & 4.55 & 4.61 & 4.53 \\
12 & 4.6 & 4.61 & 4.55 & 4.61 & 4.55 \\
14 & 4.65 & 4.61 & 4.6 & 4.62 & 4.57 \\
\hline
\end{tabular}

Tabla 9, se muestran los resultados de la acidez titulable para las diferentes formulaciones. Donde la acidez fue disminuyendo debido a que la manzana continúa sus actividades bioquímicas y fisiológicas en el cual involucran; el ablandamiento, como cambios en la firmeza y en la velocidad de respiración, entre otros. 
Tabla 9. Registro de acidez titulable en manzanas Red Delicious con RC en diferentes formulaciones

\begin{tabular}{cccccc}
\hline \multirow{2}{*}{ Días } & \multicolumn{5}{c}{ Acidez Titulable } \\
\cline { 2 - 6 } & Patrón & F1 & F2 & F3 & F4 \\
\hline 0 & $0.20 \%$ & $0.20 \%$ & $0.20 \%$ & $0.20 \%$ & $0.20 \%$ \\
2 & $0.19 \%$ & $0.20 \%$ & $0.20 \%$ & $0.20 \%$ & $0.20 \%$ \\
4 & $0.17 \%$ & $0.18 \%$ & $0.18 \%$ & $0.18 \%$ & $0.19 \%$ \\
6 & $0.17 \%$ & $0.17 \%$ & $0.18 \%$ & $0.18 \%$ & $0.19 \%$ \\
8 & $0.17 \%$ & $0.17 \%$ & $0.17 \%$ & $0.17 \%$ & $0.18 \%$ \\
10 & $0.16 \%$ & $0.16 \%$ & $0.15 \%$ & $0.15 \%$ & $0.17 \%$ \\
12 & $0.15 \%$ & $0.16 \%$ & $0.15 \%$ & $0.15 \%$ & $0.17 \%$ \\
14 & $0.12 \%$ & $0.15 \%$ & $0.15 \%$ & $0.15 \%$ & $0.16 \%$ \\
\hline
\end{tabular}

En la Tabla 10, se evidencia el comportamiento del índice de madurez en las manzanas Red Delicious con RC en diferentes formulaciones. El cual esta está relacionada con el aumento del pH y la disminución del porcentaje de acidez con el proceso de maduración.

Tabla 10. Registro de índice de madurez en manzanas Red Delicious con RC en diferentes formulaciones

\begin{tabular}{clllll}
\hline \multirow{2}{*}{ Días } & \multicolumn{5}{c}{ Índice de madurez } \\
\cline { 2 - 6 } & Patrón & F1 & F2 & F3 & F4 \\
\hline 0 & 55 & 55 & 55 & 55 & 55 \\
2 & 58 & 55 & 55 & 55 & 55 \\
4 & 72 & 61 & 64 & 64 & 61 \\
6 & 74 & 68 & 64 & 64 & 61 \\
8 & 74 & 71 & 69 & 69 & 67 \\
10 & 80 & 78 & 80 & 80 & 71 \\
12 & 87 & 78 & 81 & 83 & 72 \\
14 & 113 & 87 & 83 & 83 & 77 \\
\hline
\end{tabular}

Tabla 11, se observa la evaluación microbiológica de manzana red delicious con RC, con diferentes formulaciones despues de 15 días de alamacenamiento.

Tabla 11. Criterios microbiológicos en manzanas Red Delicious con RC en sus diferentes formulaciones después de 15 de

\begin{tabular}{|c|c|c|c|c|c|c|c|c|c|c|}
\hline \multirow{2}{*}{ Agente microbiano } & \multirow{2}{*}{ Categoría } & \multirow{2}{*}{ Clase } & \multirow[b]{2}{*}{$\mathbf{N}$} & \multirow{2}{*}{$\mathrm{C}$} & \multicolumn{2}{|c|}{ Límite por g. } & \multicolumn{4}{|c|}{ Resultado } \\
\hline & & & & & $\mathbf{m}$ & $\mathbf{M}$ & T1 & T2 & T3 & T4 \\
\hline $\begin{array}{l}\text { Aerobios Mesófilos } \\
\qquad\left(30^{\circ} \mathrm{C}\right)\end{array}$ & 2 & 3 & 5 & 2 & $10^{4}$ & $10^{6}$ & $1.7 \times 10^{1}$ & $\begin{array}{c}1.7 \\
\times 10^{1}\end{array}$ & $1.7 \times 10^{1}$ & $\begin{array}{c}1.7 \\
\times 10^{1}\end{array}$ \\
\hline Escherichia coli & 6 & 3 & 5 & 1 & 10 & $10^{2}$ & $<3$ & $<3$ & $<3$ & $<3$ \\
\hline Mohos & 8 & 3 & 5 & 1 & $<10$ & 10 & $0.4 \times 10^{1}$ & $0.9 \times 10^{1}$ & $0.9 \times 10^{1}$ & $0.9 \times 10^{1}$ \\
\hline Levaduras & 5 & 3 & 5 & 2 & $<50$ & 50 & Ausencia & $0.2 \times 10^{1}$ & $0.2 \times 10^{1}$ & $0.2 \times 10^{1}$ \\
\hline Salmonella sp. & 10 & 2 & 5 & 0 & Aus.25.g & --- & $\mathbf{0}$ & $\mathbf{0}$ & $\mathbf{0}$ & $\mathbf{0}$ \\
\hline
\end{tabular}


Tabla 12. Resultados de la evaluación sensorial

\begin{tabular}{|c|c|c|c|}
\hline \multicolumn{4}{|c|}{ Rango para el Color } \\
\hline Muestras & Casos & Media & Grupos Homogéneos \\
\hline M4 & 30 & 2.8 & a \\
\hline M3 & 30 & 2.83333 & a \\
\hline M2 & 30 & 4.73333 & $\mathrm{~b}$ \\
\hline M1 & 30 & 4.73333 & $\mathrm{~b}$ \\
\hline \multicolumn{4}{|c|}{ Rangos para el Olor } \\
\hline M3 & 30 & 2.7 & $\mathrm{a}$ \\
\hline M4 & 30 & 2.8 & a \\
\hline M2 & 30 & 4.5 & $\mathrm{~b}$ \\
\hline M1 & 30 & 4.63333 & $\mathrm{~b}$ \\
\hline \multicolumn{4}{|c|}{ Rangos para la Textura } \\
\hline M4 & 30 & 2.43333 & $\mathrm{a}$ \\
\hline M3 & 30 & 2.73333 & a \\
\hline M2 & 30 & 3.56667 & $\mathrm{~b}$ \\
\hline M1 & 30 & 3.76667 & $\mathrm{~b}$ \\
\hline \multicolumn{4}{|c|}{ Rangos para la apariencia } \\
\hline M4 & 30 & 2.9 & $\mathrm{a}$ \\
\hline M3 & 30 & 3.03333 & a \\
\hline M2 & 30 & 4.5 & $\mathrm{~b}$ \\
\hline M1 & 30 & 4.63333 & $\mathrm{~b}$ \\
\hline \multicolumn{4}{|c|}{ Rangos para el sabor } \\
\hline M4 & 30 & 2.3 & $\mathrm{a}$ \\
\hline M3 & 30 & 2.73333 & $a b$ \\
\hline M2 & 30 & 3.03333 & $\mathrm{~b}$ \\
\hline M1 & 30 & 4.63333 & C \\
\hline \multicolumn{4}{|c|}{ Rangos para el agrado } \\
\hline M4 & 30 & 1.96667 & $\mathrm{a}$ \\
\hline M3 & 30 & 2.5 & b \\
\hline M2 & 30 & 3.23333 & c \\
\hline M1 & 30 & 4.56667 & d \\
\hline
\end{tabular}

Nota: letras iguales indican que no existe diferencias estadísticamente significativas., y letras diferentes indican que existe diferencias estadísticamente significativas

\section{Evaluacion sensorial de manzana red delicious RC}

En la Tabla 12, se muestra en análisis estadístico mediante la prueba de Tukey de la evaluación sensorial para el color, olor, textura, apariencia, sabor y agrado (Después de dos semanas de almacenamiento). Donde la prueba para el sabor se distingue por presentar el mejor color rojo brillante característico de la manzana red Delicious. Para el olor en la muestra M3 y M4, presentaron el mejor olor aromático. En la textura y la apariencia las mejores muestras correspondieron a M3 y M4 por tener mejor firmeza en la textura y mejor apariencia. Con respecto al sabor se puede observar que hay 
diferencias significativas en la muestra M3, siendo la muestra de mejor sabor la M1. Así mismo el rango del agrado se evidencia la mejor muestra fue M1.

\section{DISCUSIÓN}

Los grados ${ }^{\circ}$ Brix del mucílago de café fresco varían según el estado de maduración del café cerezo. En promedio, el mucilago del café pintón contiene $14.1^{\circ} \mathrm{Brix}$, el maduro $17.1^{\circ} \mathrm{Brix}$, y el sobre maduro $20.1^{\circ}$ Brix. Los grados ${ }^{\circ}$ Brix del mucilago de café fresco son un indicador de la madurez del grano despulpado y los valores de pH del mucílago fermentado entre 3.7 y 4.1 son adecuados y seguros para interrumpir la fermentación y lavar el café (Puerta, 2012).

El método más común para determinar el contenido de humedad es a través de la pérdida de peso mediante el método de secado en mufla o estufa, donde el contenido de humedad se determina a partir del cambio de peso de la muestra después de la evaporación del agua absorbida en el horno (Austin et al., 2013). Teniendo en cuenta que la determinación de humedad por el método de mufla es un método muy extenso ya que en el protocolo existen actividades que pueden afectar dicha determinación como pueden ser la tara de crisoles, la manipulación de la muestra y el medio ambiente, los cuales son factores que influyen directamente en la determinación del porcentaje de humedad ocasionando mediciones del peso incorrectos (Sánchez, 2007). Los porcentajes de humedad obtenidos del RC van desde 9.7\% 11.3\%, mostrando valores más bajos las formulaciones que presentan mayor porcentaje de Gelatina en su formulación (F3 y F4) debido al incremento de sólidos solubles. Estos valores guardan relación con los encontrados por Moncayo (2013), quien encontró porcentaje de humedad entre los rangos de 9.4\% a 15.4\%, las diferencias encontradas en su investigación se deben que existen diferencias significativas en el espesor al usar sorbitol o glicerol y al adicionar pectina, posiblemente por la estructura molecular de cada una, su efecto plastificante y el incremento de sólidos en la formulación.

Según Robles (s.f.) afirma que el gramaje está directamente relacionado con la resistencia e intensidad de trasmisión de luz de los materiales. Un mejor gramaje ofrece una mejor resistencia mecánica y una mejor barrera a la transmisión de luz. Teniendo en cuenta estos mecanismos, los films y recubrimientos deben cumplir una serie de requerimientos para poder garantizar su efectividad y funcionalidad. Algunos de estos requerimientos son propiedades adecuadas para actuar como barrera, que mantenga el color y la apariencia del alimento, que garantice una serie de características mecánicas y comportamientos reológicos, y que se trate de compuestos no tóxicos para el ser humano (Dehghani et al., 2017; Ramos et al., 2012). 
El grado ${ }^{\circ}$ Brix, pH, acidez titulable e índice de madurez están dentro de los rangos establecidos por la OSCE. Con respecto al valor del pH obtenido y su porcentaje de acidez, son valores que se ajustan a la evaluación sensorial, teniendo en cuenta que esta variedad de manzana presenta un sabor dulce, con poco ácido y es un alimento que está dentro de la clasificación de los productos poco ácidos. De la evaluación organoléptica se determinó parámetros como el sabor dulce, olor característico, color rojo brillante, apariencia lisa y textura firme, datos importantes para realizar la evaluación de las manzanas con RC, en sus diferentes formulaciones durante el periodo de almacenamiento. Los datos encontrados guardan relación con la ficha técnica de la OSCE, para manzana Red Delicious en su estado óptimo de comercialización. Las manzanas son frutas climatéricas que continúan madurando después de separadas del árbol. Para su almacenamiento a largo plazo es mejor cosechar las manzanas antes del aumento climatérico de las tasas de respiración y producción de etileno. La aplicación de las tecnologías apropiadas para mantener la calidad depende del conocimiento de la estructura del fruto, la fisiología y las transformaciones metabólicas (Pech et al., 2013).

Las pérdidas de peso (\%PP) fueron determinadas por gravimetría, mediante el registro de los pesos de cada manzana con RC en las diferentes concentraciones. Los resultados nos muestran que la aplicación de RC nos ayuda a reducir de forma gradual la pérdida de peso. Los recubrimientos reducen la transferencia de vapor de agua (Dhall, 2013), además sellan pequeñas fisuras en el tejido. En comparación con los recubrimientos comestibles simples, los recubrimientos compuestos preservan de mejor manera la turgencia y reducen la pérdida de peso de las frutas (Han, 2014). Los resultados obtenidos están relacionados con los presentados por del Valle et al. (2005), quienes trabajaron con mucilago de cactus en fresas, así como los de Yaman et al. (2002), quienes trabajaron con un recubrimiento comercial a base de polisacáridos y ácidos grasos en cerezas; ambos han obtenido reducciones significativas en la pérdida de peso.

La utilización del RC ayuda a reducir la continuidad del proceso de maduración de la manzana, por lo tanto, retrae la producción de solidos totales, hecho que no ocurre en el tratamiento patrón donde se muestra una producción mayor de solidos solubles. Se observa que entre el día 0 y el día 14 hubo un aumento progresivo en los valores de SST en el tratamiento patrón al variar de $11^{\circ}$ Brix a $13.5^{\circ}$ Brix. Este aumento es debido a la hidrólisis de almidones propio del proceso de maduración de frutos climatéricos como la manzana. Esta tendencia podría estar relacionada con el retardo del proceso de maduración en las muestras con recubrimiento, debido a su acción protectora, comportamiento observado anteriormente por Castricini (2009). 
Los resultados del pH fueron afectados por el uso de RC, está relacionado con la disminución de la senescencia del fruto, evitando que durante la maduración de la manzana algunos fragmentos de pectinas se liberen desde la pared celular y se unan a los polifenoles, lo cual incrementa los valores de pH. González, (2010), argumenta que un aumento en los valores de $\mathrm{pH}$ durante el almacenamiento demuestra el proceso de senescencia del producto. La disminución de la acidez, pudo ser debido a que la manzana continua sus actividades bioquímicas y fisiológicas en el cual involucran; el ablandamiento, como cambios en la firmeza y en la velocidad de respiración, entre otros; son irreversibles una vez iniciadas, su velocidad se podría retrasar debido a la aplicación de otros tratamientos (Omboki et al., 2015).

\section{CONCLUSIONES}

Los recubrimientos comestibles obtenidos presentaron características fisicoquímicas (\% de humedad, gramaje, espesor y peso específico) dentro de los rangos establecidos para los recubrimientos comestibles aplicados en la conservación de frutas mínimamente procesadas. Los resultados obtenidos indicaron que las aplicaciones del recubrimiento comestible en sus 4 formulaciones permiten reducir la pérdida de peso; controlar el aumento del ${ }^{\circ}$ Brix, pH e índice de madurez y ayudan a la disminución de la acidez titulable. Por último, la formulación F1, es la que presentó mejor aceptación según la Prueba de Tukey a un nivel de confianza del 95\%. Siendo que esta formulación no altera las características sensoriales. El recubrimiento comestible a partir de mucilago de café mejora la calidad del producto tratado, retrasan la maduración y deterioro de los mismos, preservando de tal forma sus atributos comerciales y alimenticios.

\section{REFERENCIAS BIBLIOGRÁFICAS}

Allegra, A., Inglese, P., Sortino, G., Settanni, L., Todaro, A. and Liguori, G. 2016. The influence of Opuntia ficus-indica mucilage edible coating on the quality of "Hayward” kiwifruit slices. Postharvest Biology and Technology, 120, 45-51.

Austin, J. y otros tres autores, Utilizing microwaves for the determination of moisture content independent of density, Powder Technology: 236 (Special Issue), 17-23 (2013).

Castricini, A. 2009. Aplicação de revestimentos comestíveis para conservação de mamões (Carica papaya L.) 'Golden’. Tese Doutorado en Fitotecnia. Universidade Federal Rural do Rio de Janeiro, Departamento de Fitotecnia, Instituto de Agronomia. 110 p. 
Corbo, MR, Campaniello D,Speranza B,Bevilacqua A, and Sinigaglia M. 2015. NonConventional Tools to Preserveand Prolongthe Quality of Minimally-Processed Fruits and Vegetables. (Articulo Coatings, 5, 931-961; doi: 10.3390/coatings5040931) (Google Academico).

Dhall, R. (2013). Advances in Edible Coatings for Fresh Fruits and Vegetables: A Review. Critical Reviews in Food Science and Nutrition, 53(5), 435-450.

Ancos, B., Gonzalez-Peña, D., Colina-Coca,C., Sánchez-Moreno, C.2015.Uso de Películas/Recubrimientos Comestibles en los Productos de IV y V Gama Revista Iberoamericana de Tecnología Postcosecha, Vol. 16, (1), pp. 8-17.

Dehghani, S., Hosseini, S. V. y Regenstein, J. M. (2017). Edible films and coatings in seafood preservation : A review. Food Chemistry, 240, 505-513. Recuperado de: http://dx.doi.org/10.1016/j.foodchem.2017.07.034

Fai, A. E. C., de Souza, M. R. A., de Barros, S. T., Bruno, N. V., Ferreira, M. S. L., and de Andrade Gonçalves, É. C. B. (2016). Development and evaluation of biodegradable films and coatings obtained from fruit and vegetable residues applied to fresh-cut carrot (Daucus carota L.). Postharvest Biology and Technology, 112, 194-204.

González, M., 2010. Conservación de mora, uvilla y frutilla mediante la utilización del aceite de canela (Cinnamomum zeynalicum), Proyecto de titulación previo a la obtención de Bioquímico Farmacéutico, ESPOCH, Riobamba, Ecuador p. 121.

Han, J. (2014). Edible Films and Coatings. En J. Han (Ed.), Innovations in Food Packaging (Second Edition., pp. 213-255). Plano, TX, Estados Unidos: Elsevier.

Moncayo, C. (2013). Desarrollo de un recubrimiento comestible a partir de un biopolímero para prolongar la vida útil de frutas frescas. Tesis para optar el grado de Master en ciencia y tecnología de alimentos. Universidad nacional de Colombia. Bogotá, Colombia.

Omboki, R. B.; Wu, W.; Xie, X. and Mamadou, G. 2015. Ripening genetics of the tomato fruit.Inter. J. Agric. Crop Sci.8 (4):567- 572.

Pech, J. C.; Purgatto, E.; Girardi, C. L.; Rombaldi, C. V. and Latché, A. 2013. Current challenges in postharvest biology of fruit ripening. Current Agric. Sci. Technol. 19(1-18).

Puerta Q., G.I.; Ríos a., s. 2011. Composición química del mucílago de café, según el tiempo de fermentación y refrigeración. Revista de Cenicafé 62 (2): 23-40. (Google Académico). 
Puertas-Mejía, Miguel A, Villegas-Guzmán, Paola, \& Alberto Rojano, Benjamín. (2013). Borra de café colombiano (Coffea arabica) como fuente potencial de sustancias con capacidad antirradicales libres in vitro. Revista Cubana de Plantas Medicinales, 18(3), 469-478.

Rathinavelu. R y Graziosi G., 2005. Posibles usos alternativos de los residuos y subproductos del café. En ICS-UNIDO, Science Park, Padriciano, Trieste, Italia; Departamento de Biología de la Universidad de Trieste (Italia).

Ramos, O. L., Fernandes, J. C., Silva, S. I., Pintado, M. E. y Malcata, X. (2012). Edible Films and Coatings from Whey Proteins: A Review on Formulation, and on Mechanical and Bioactive Properties. Critical Reviews in Food Science and Nutrition, 52, 533-552. DOI: $\underline{10.1080 / 10408398.2010 .500528}$

Robles, J. (s.f.). Control de Calidad de Envases y Embalajes de Alimentos. Facultad de Ingeniería. Universidad Privada Antonio Guillermo Urrelo. Cajamarca - Perú.

Rojas Graü Maria A. "Recubrimiento y sustancias de origen natural en manzana fresca cortada: Una nueva estrategia de conservación.” Tesis de doctorado. 58 Departamento de Tecnología de Alimentos. Universidad de Lleida. España, 2006.

Rodriguez, S. "Desarrollo y Caracerizacion de una Pelicula Funcional a base de Goma Guar y Aceite de Oliva” “Universidad Autonoma Agraria Antonio Narro”. México, 2016.

Sánchez, A., Estudio comparativo de los métodos empleados para la determinación de humedad (mufla y analizador de humedad de halógenos) aplicado a producto cárnico tipo salchichón, mortadela, jamón y chorizo, Tesis de pregrado, Facultad de Ingenierías y Arquitecturas, Universidad de Pamplona, Pamplona, Colombia (2007).

Yaman, I.O., Hearn, N. \& Aktan, H.M. Active and non-active porosity in concrete Part I: Experimental evidence. Mat. Struct. 35, 102 (2002). https://doi.org/10.1007/BF02482109 .

Copyright $($ de los autores. Titular de la licencia: Revista Pakamuros. Este artículo es un artículo de acceso abierto distribuido bajo los términos y condiciones de la licencia Creative Commons (CC BY-NC) (https://creativecommons.org/licenses/by-nc/4.0/). 